\title{
Remote Haptic Feedback from a Dynamic Running Machine
}

\author{
Hanns Tappeiner, Sarjoun Skaff, Tomas Szabo and Ralph Hollis
}

\begin{abstract}
In this paper we present our efforts to design a system for feeding back useful haptic information from a highly dynamic running robot to a remote operator using a haptic device. Without adding additional sensors, the legs of the robot are used as feelers to give the operator the capability to both explore and manipulate the robot's environment and to gather meaningful information about properties not captured by visual feedback like weight, movability and structure of an encountered object. We show the capabilities of the system in a user study with both trained and untrained operators.
\end{abstract}

\section{INTRODUCTION}

Teleoperation of robots has shown the benefits of combining the inherent physical capabilities of the machine with the cognitive capabilities of the human [1], [2]. For the past several decades, teleoperation of mobile robots has incorporated joysticks, switch closures, and other simple mechanisms for commanding the machine, combined with visual feedback from an on-board video camera to help guide the operator. Recently, however, there has been a growing recognition that teleoperation efficacy can be markedly improved by the addition of haptic feedback. Haptic control of mobile robots is not an entirely new topic. This paper, unlike previous work, in addition to visual feedback via a wireless camera, concerns haptic feedback from a legged mobile robot. Using the legs as feelers gives an operator the capability to further explore and manipulate the environment of the robot while providing haptic feedback.

The implemented system was tested on operators, some familiar with using a haptics device and some not, to evaluate their performance in exploring a remote environment. Examples of tasks performed include distinguishing between objects of different masses, and between movable or non-movable objects like doors that are ajar or locked. These are tasks which are difficult or

This work was supported in part by NSF grant IIS-0413085.

$\mathrm{H}$. Tappeiner is a PhD student at the Robotics Institute, Carnegie Mellon University, Pittsburgh, PA, USA hanns @ cmu . edu

Sarjoun Skaff is a postdoctoral fellow at the Robotics Institute, Carnegie Mellon University, Pittsburgh, PA, USA sarjoundemu.edu

T. Szabo is a student at Technical University of Munich, Munich, Germany, tomas.szabo@gmx.de

R. Hollis is a research professor at the Robotics Institute, Carnegie Mellon University, Pittsburgh, PA, USA rhollis@ cs . cmu . edu

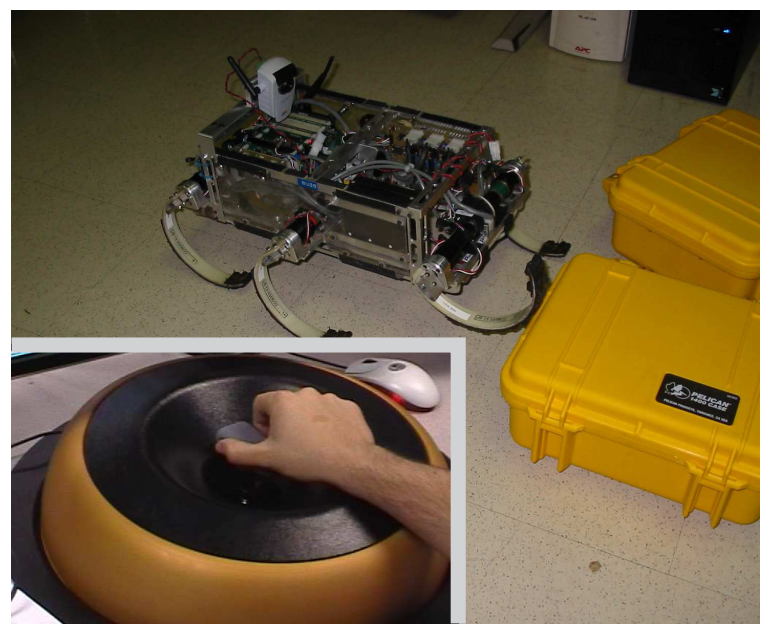

Fig. 1. RHex using its front right leg to touch and move a plastic box. Using the haptic feedback from the leg, the operator determines whether the box can be moved or is too heavy to move and its relative weight.

even impossible to perform with pure visual feedback alone.

\section{A. Motivation}

As a dynamic running machine, RHex [16], [17] has shown the ability to traverse different kinds of extremely rough terrain, which make the robot ideal, e.g., for search and rescue tasks in both urban and outdoor environments. Instead of adding special manipulators to the robot, we propose that RHex's uniquely shaped legs can also be used as manipulators to carefully explore and manipulate objects of interest and that haptic feedback from those legs will increase the operator's ability to understand the robot's remote environment. This would reduce both complexity and weight of the robot to a minimum as well as increase ruggedness.

\section{RELATED WORK}

The work of Barnes and Counsell in 1999 is one of the earliest efforts to integrate haptic feedback in the control of mobile robots [3]. Several experiments were performed which showed improved operator performance when haptics was used. Khatib, et al., describe efficient algorithms supporting haptic interaction with realistic 
physical models of mobile robots [4]. Rosch, et al., used a force-feedback joystick to control a small mobile robot equipped with force sensors [5]. Park, et al., consider the teleoperation of a mobile robot for hazardous environment applications using a distance-based repulsive force for obstacle avoidance [7] with a novel 6-DOF haptic master [8]. Diolaiti and Melchiorri demonstrated haptic teleoperation of a small Activmedia Pioneer differentialdrive mobile robot using a 3-DOF PHANToM haptic interface [9], [10]. Lee, et al., provide one of the most complete descriptions of haptic teleoperation of a mobile robot [11]. The authors adopt a "car-driving" metaphor that maps the position of a 2-DOF haptic device to the speed and turning rate of a car-like mobile robot. Results were analyzed both objectively and subjectively, indicating a statistically significant positive benefit for the inclusion of haptic feedback in the teleoperated control of the robot.

The system described in this paper, is more comparable to a mobile platform equipped with various manipulators which allow the operator to specifically gather information about the robot's environment and to manipulate it. This is done without actually adding any manipulators to the robot or even any additional sensors. The robot's six legs are used as tactile feelers.

\section{SYSTEM OVERVIEW}

\section{A. The running machine RHex}

RHex is a six-legged robot (Fig. 2) that can execute a collection of dynamic behaviors, including walking, jogging, and running. These behaviors are made possible by synchronizing the legs three by three to produce an alternating tripod gait, and by designing the legs to have specific compliance properties. Compliance allows the legs to store and release energy in the form of elastic deformation, thus enabling energy-efficient locomotion. These characteristics are particularly important for producing jogging and running behaviors which alternate flight and stance phases akin to animal running, and propel the body at speeds up to five body lengths per second [16], [17]. The machine has an on-board video camera and various on board sensors like an IMU. In the usual case, the operator observes the robot visually directly or via the on-board camera while commanding forward/backward speed and turning rate. The robot's ability to run and scramble through forests, rubble, mud, and other harsh terrain is unprecedented. With a gear ratio of 33:1 and a moment arm (leg length) of $0.21 \mathrm{~m}$ from center of rotation to the tip, the single legs are highly backdrivable (from the tip of the leg). As we will see later, this makes them well suited for

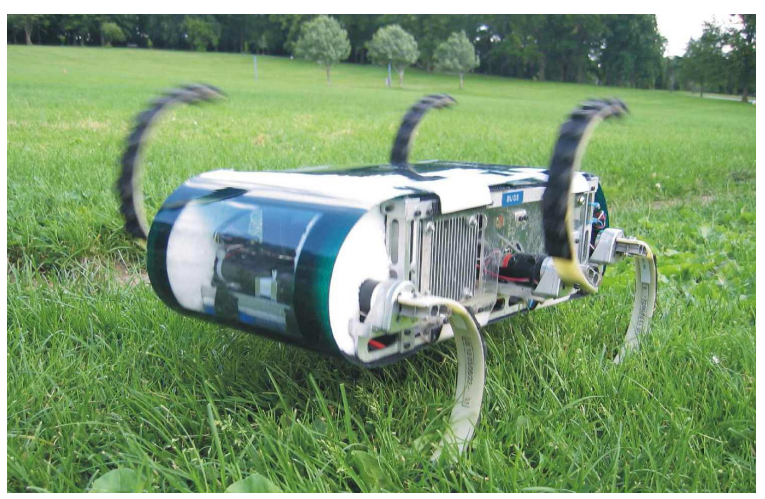

Fig. 2. RHex running outdoors on a grassy surface in a standard tripod gait.

measuring torque to generate tactile feedback without adding additional sensors. Another important property of the robot is the fact that it can stand very stably on five or even only four of its legs, so that any one or two legs can be used as haptic feelers.

\section{B. The Maglev Haptics Interface}

TABLE I

ABBREVIATED PERFORMANCE CHARACTERISTICS OF 2ND GENERATION MAGNETIC LEVITATION HAPTIC INTERFACE SYSTEMS.

\begin{tabular}{|l|l|}
\hline Attribute & Value \\
\hline \hline Degrees of freedom & 6 \\
Maximum impedance & $50.0 \mathrm{~N} / \mathrm{mm}$ \\
Spherical workspace & $24 \mathrm{~mm}$ diameter \\
Minimum impedance & $0.002 \mathrm{~N} / \mathrm{mm}$ \\
Rotation range & $\pm 8^{\circ}$ \\
Position bandwidth & $140 \mathrm{~Hz}(-3 \mathrm{~dB})$ \\
Position resolution & $2 \mu \mathrm{m}(1 \sigma)$ \\
\hline
\end{tabular}

The haptic master system features a newly developed desktop-mounted magnetic levitation haptic device (MLHD) shown in Fig. 3. The device has a light weight bowl-shaped "flotor" containing six spherical coils that is levitated in strong magnetic fields created by $\mathrm{NdFeB}$ permanent magnets. An interchangeable handle is rigidly attached to the flotor. Three LEDs attached to the flotor are tracked by optical position sensors, thereby enabling closed-loop control. The device exhibits extremely high fidelity since there are no motors, gears, bearings, cables, or linkages present as in conventional haptic devices. Its high stiffness range and frequency response characteristics not only engage the user's proprioceptive senses, but also to a significant degree the touch sensors in the skin such as the Pacinian corpuscles having a peak sensitivity at about $250 \mathrm{~Hz}$ [18]. The haptic master 


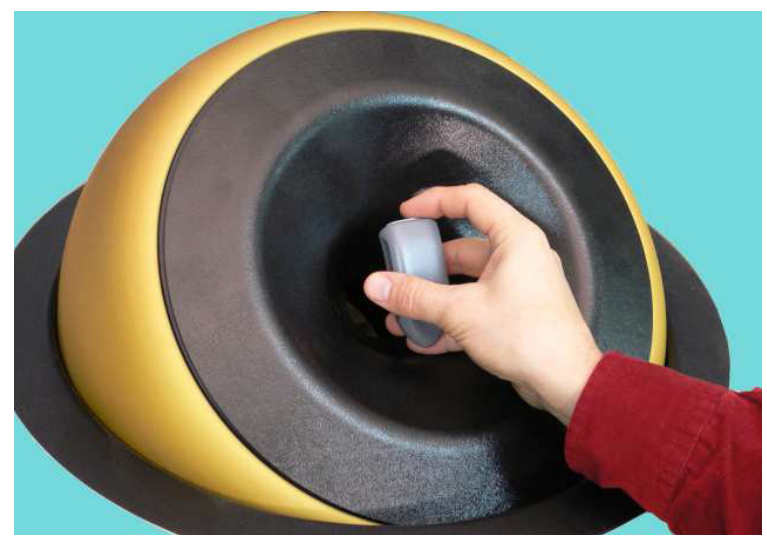

Fig. 3. New second-generation 6-DOF magnetic levitation haptic device: overall view showing the spherical swivel mounting in a desktop which allows re-orientation to suit users' preferences

behaves as an almost ideal "impedance" device, where handle positions and angles are sent to the remote robot, and pure forces and torques are displayed to the hand in return. Compared with the popular 3-DOF PHANToM haptic interface, the MLHD has a bandwidth of $140 \mathrm{~Hz}$ compared to about $10 \mathrm{~Hz}$, and maximum stiffness of $50 \mathrm{~N} / \mathrm{mm}$ compared with about $2 \mathrm{~N} / \mathrm{mm}$. The smaller motion range of the device is easily overcome by scaling, rate control, and indexing if necessary. We believe the MLHD master is an ideal device for conveying accurate, subtle, high-frequency information from teleoperated mobile robots. Table I briefly summarizes its main performance characteristics.

\section{Combined system}

The robot is connected via an $801.11 \mathrm{~b}$ wireless interface to a control computer which also serves as a host machine for the MLHD. The MLHD has its own controller running QNX. The robot's software supports various modes which allow it to perform certain tasks, e.g., a walking mode, a running mode, and a stair climbing mode. The operator decides when and how to switch between single modes. In the current setup, an operator controls RHex visually, using video from the remote robot displayed on the operators monitor. In all cases, the robot's behavior is controlled using the MLHD. Proportional movement of the haptic device handle forward, backward, or in rotation (yaw), causes RHex to walk or run forward, reverse, to rotate by changing the phasing of the legs or to pitch and roll the body. When approaching an object of interest, the operator can switch the robot into Haptic Feedback Mode and selects one or multiple legs to be used as feelers to explore and/or manipulate the object.

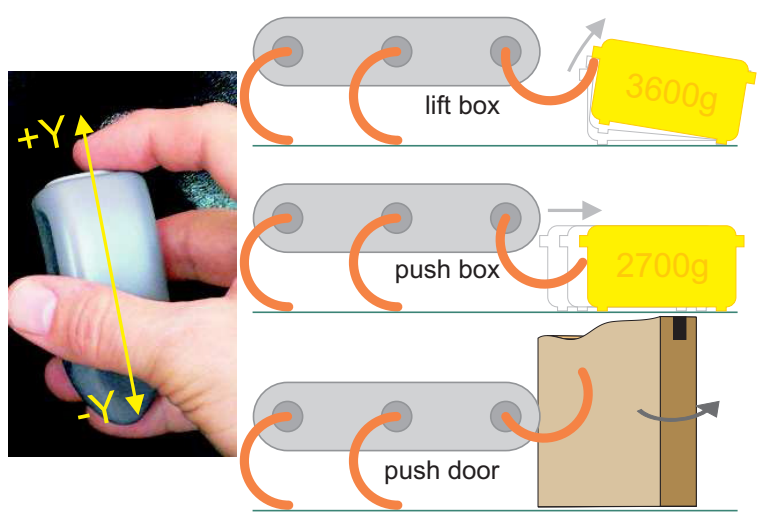

Fig. 4. Example tasks which use the haptic feedback: lifting/pushing boxes with diferent weight, distinguishing between doors ajar and locked etc.

\section{HAPTIC FEEDBACK}

One goal of the system setup was the necessity of not having additional sensors on the robot. Each RHex leg is driven by a Maxon 118751 electric motor, the gear ratio of the planetary gear is 33:1 and the length of the attached leg is $0.21 \mathrm{~m}$. A PD controller controls the leg's angular position. Since the legs are very back drivable, the position difference or proportional error $d p$ between the desired and the actual position of the legs can be used to compute the torque $t$ the motor applies to the leg in order to reach the desired position. The bigger the proportional error, the higher the torque applied to the leg. Assuming a leg usually touches an obstacle at or near the tip of the leg and knowing the length of the leg, one can compute the force the leg applies to an obstacle based on the position error. This approach ignores any friction in the joint, so higher gear ratios which have higher friction will not work as well.

For the experiments described in this paper, we only use one of the six DOF's of the MLHD: The Maglev's $y$ axis is mapped to the angular position of a selected RHex leg, shown in Fig. 3. However, it is important to realize that feeding back other haptic information derived from on-board accelerometers, multiple legs simultaneously etc. will potentially make use of all six DOFs. Also, four DOFs of the robot are being used to control the robot: in addition to simply controling the speed and steering, $x$ and $y$ orientation on the MLHD is used to control the robot's pitch and roll. It is also important to realize that the MLHD is both an input and output device. Thus to move a robot leg, the operator must move the handle in the described direction, and to experience the force derived from the remote leg 


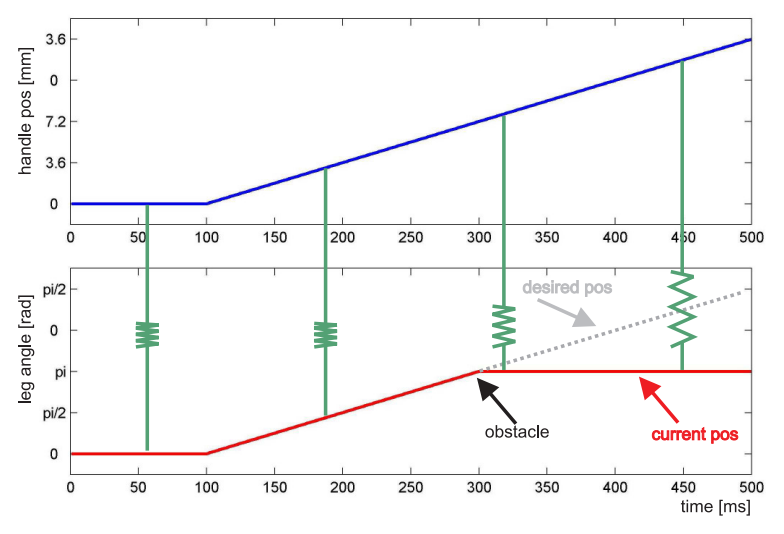

Fig. 5. Virtual coupling illustration between the haptic handle and the robots angular leg position. At $\mathrm{t}=300$ the leg hits an obstacle.

controller, the MLHD must produce a force that is opposite to the operators hand motion.

The connection between the MLHD and robot leg constitutes a servo loop that is potentially unstable. To help insure stability, a method known as virtual coupling is used as in [19]. The leg and the haptic handle are connected by a virtual spring. In theory, the leg moves exactly as commanded by the haptics device and the spring is not stretched (the difference between commanded position and leg position is 0). In case a leg touches an obstacle but the operator comands further motion, the position of the leg starts to differ from the position of the haptic handle. This causes the virtual spring to be stretched, in which case a corresponding force is applied to both the haptic handle and the leg (in opposite directions) to "pull them back together" as illustrated in (Fig. 5). When using fairly low gains for the virtual spring, the update rate can drop to about $20 \mathrm{~Hz}$ or even less. To get good haptic feedback from the robot which feels reasonably smooth, an update rate of 100 $\mathrm{Hz}$ was necessary. That is easily achivable by standard WIFI components, assuming the robot is within a few hundred meters of the operator.

\section{RESULTS}

Using the system described above, operators had to perform a series of tasks (Fig. 4). The goal was to determine real world system performance, meaning whether the system can actually generate useful haptic feedback allowing an operator to experience properties of the remote robot's environment which could not be explored by pure visual feedback from a camera. The tasks included using RHex legs via the MLHD to:

1) Push against different objects to determine whether they are movable.
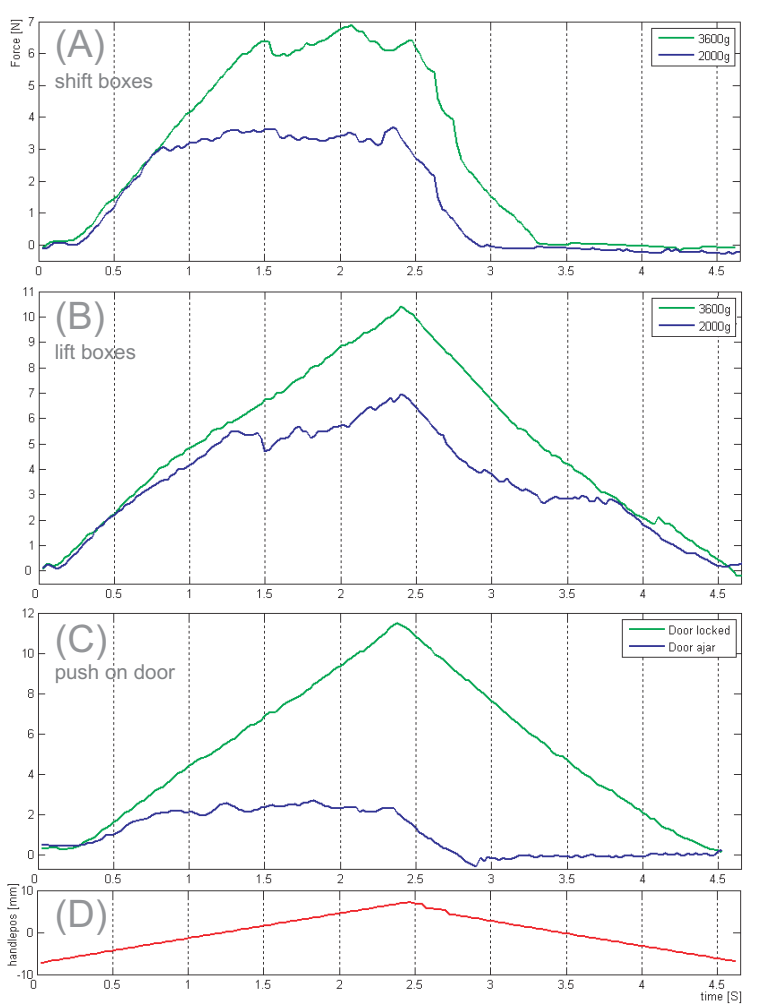

Fig. 6. Force profiles of a leg (at the tip of the leg) - mapped to the haptic device - when the leg is pushing against obstacles. A) Leg pushes plastic boxes on the floor. B) Leg lifts/tilts the boxes C) Leg pushes against a door which is ajar or locked. D) Shows the corresponding position of the haptics device handle/ proportional to the leg angle.

2) Push against different objects to determine their relative weights.

3) Carefully manipulate objects like lifting boxes if they are not too heavy or try to open doors if they are ajar and not locked.

All tests were performed with the robot out of sight of the operator. During the actual force feedback task, all visual feedback, including the camera image and the sensor data display, was turned off. The only feedback was force fed back to the haptic device.

\section{A. Force profiles}

Fig. 6 shows plots of three sets of force profiles recorded during the experiment. (A) shows the profile from the front right leg shifting two identical-looking yellow plastic cases on the floor. The task was for the operator to determine which box was the heavier one. The green curve corresponds to a case with a mass of $3.6 \mathrm{~kg}$, the blue curve to a case with a mass of 2.0 $\mathrm{kg}$. Graph (B) shows the profiles when manipulating the same two boxes, but instead of shifting them along the 
ground, the leg was used to lift/tilt the boxes. Again, the green plot comes from the $3.6 \mathrm{~kg}$ box and blue from the $2.0 \mathrm{~kg}$ box. (C) shows the force profiles from the right front leg used to push against a door. The task was to determine whether the door is locked or just ajar and can be passed through. The green curve was obtained from pushing against a closed door, the blue one from pushing against a door that was ajar. The forces are given in Newtons [N] and correspond to the forces the legs would apply to an object when pushing with the tip of the leg. Graph (D) shows the position of the haptic device handle in [mm] moving from $-7.2 \mathrm{~mm}$ to $7.2 \mathrm{~mm}$. (D) corresponds to the force profiles in (A), (B) and (C). To generate comparable force profile plots for Fig. 6, the handle was automatically moved at constant velocity.

\section{B. User study}

To test whether operators really can distinguish between objects with different masses purely based on the haptic feedback from a RHex leg, we performed a small series of experiments with six operators. Three of the operators were familiar with the MLHD, and three were not.

The subjects each completed 9 experiments where they pushed against a heavier and then a lighter box or vice versa and were required to judge which one was heavier. The order of heavy and light was chosen at random. The operators had no test trials, meaning they didn't have the chance to get used to the system before the experiment. The first trial was already part of the experiment.

The first four experiments were performed with a $2.0 \mathrm{~kg}$ box (light) and a $3.6 \mathrm{~kg}$ box (heavy), the next four experiments with a $2.0 \mathrm{~kg}$ box (light) and a $27 \mathrm{~kg}$ box (heavy). The 9 th experiment was a trick question where the $2.0 \mathrm{~kg}$ box was used twice. This last trial wasn't meant to produce relevant data but just to see how operators would react. Table II shows the results for experiments 1-8 for each operator.

TABLE II

TRIAL RESULTS FOR 6 OPERATORS WITHOUT VISUAL FEEDBACK

\begin{tabular}{|l|l|l|}
\hline Operator & 2.0 kg vs. 3.6 kg & 2.0 kg vs. 2.7 kg \\
\hline \hline 1 & $4 / 4$ & $4 / 4$ \\
2 & $4 / 4$ & $3 / 4$ \\
3 & $4 / 4$ & $4 / 4$ \\
4 & $4 / 4$ & $4 / 4$ \\
5 & $4 / 4$ & $4 / 4$ \\
6 & $4 / 4$ & $3 / 4$ \\
\hline
\end{tabular}

In the case of the $2.0 \mathrm{~kg}$ box vs. the $3.6 \mathrm{~kg}$ box, none of the operators made a mistake. In the very first experiment, it took most operators longer to judge and they usually used the whole workspace of the MLHD to explore the box, meaning they pushed the box as long as possible with the robot's leg. In the subsequent trials, operators got much quicker at judging a box's weight up to a point where they answered the question of which box was heavier almost immediately after touching the second box.

In case of the $2.0 \mathrm{~kg}$ box vs. the $2.7 \mathrm{~kg}$ box, operators made errors two out of twenty-four times. In both cases, the operators made the mistake during their first trial with $2.0 \mathrm{~kg}$ vs. the $2.7 \mathrm{~kg}$ box. Like before, the first trial took operators longer than subsequent ones.

For the trick question where we used the $2.0 \mathrm{~kg}$ box twice but didn't tell the test subjects that that was an option, all six operators took a long time to answer realtive to the earlier trials. Three operators asked whether they can try again, two said they are not sure and one said that the boxes felt the same.

\section{CONCLUSION}

The results from the experiments with different operators are very encouraging and show that operators, even without any training, are able to use the system to intuitively gather information about the remote robot's environment. This information, like object's masses and their movability, would not be available to an operator from using pure visual feedback from a camera. Even if one could potentially not feed the haptic information to the operator through the MLHD, but instead show the forces as textual or graphical data on the operator's monitor, using the haptic system seems a) immediately intuitive and b) allows the operator to constantly look at and focus on the camera image.

Operators were able to perform very well in the given tasks. Especially interesting is the fact that operators could determine whether an obstacle is light enough to be moved out of the way by the robot to clear a path. This capability might prove useful duing a search and rescue missions where an otherwise traversable path is blocked. A specific example would be a collapsed building where an operator uses the robot to search for people. The entrance to a room is blocked by several obstacles like broken furniture, bricks, etc. Using the haptic feedback, the operator can immediately test whether some of the obstacles can be moved out of the way.

\section{FUTURE WORK}

\section{A. Multi-DOF haptic feedback and detailed user study}

We are interested in applying the described idea of sensorless haptic feedback to different legged robots, e.g., those with more degrees of freedom per leg, and 


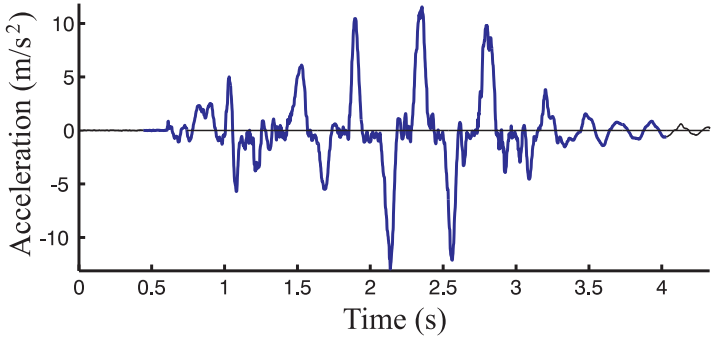

Fig. 7. Shows z-acceleration over time from the RHex on board IMU.

to see whether operators can perform complex manipulation tasks. The results of the user study are pretty clear: operators are able to perform the proposed tasks very well. But it would be very interesting to explore in detail where performance of operators drops, e.g., how similar the weights of two boxes can be until the average operator cannot distinguish between them anymore and whether in that case a trained operator would perform better than an untrained one. Those weights could be compared to corresponding weights when operators can physically touch the boxes with their hands.

\section{B. Haptic feedback for dynamic motions}

We are interested in exploring haptic feedback during tasks where the robot is not statically stable and whether the right kind of haptic feedback can increase an operator's ability to control the robot at high speeds and on rough terrain. Fig. 7 shows the acceleration along the $\mathrm{z}$ axis measured by the robot's on board IMU during slow running. Potentially useful haptic feedback could come from these types of acceleration and body attitude data. In the current setup, the wireless connection to/from the robot has a very large latency, sometimes dropping to less than 20 force feedback commands per second. Especially at high speeds, this is not enough to provide meaningful real time feedback. One of our goals is to improve the communication significantly, which is definitely possible using new WIFI components.

\section{REFERENCES}

[1] J. Vertut and P. Coiffet, Robot Technology, Vol. 3A: Teleoperations and Robotics: Evolution and Development, ser. PrenticeHall Series on Robot Technology. Prentice-Hall, 1986.

[2] T. Sheridan, Telerobotics, Automation and Human Supervisory Control. MIT Press, 1992.

[3] D. P. Barnes and M. S. Counsell, "Haptic communication for remote mobile manipulator robot operations," in Proc. American Nuclear Society 8th Topical Meeting on Robots and Remote Systems, Pittsburgh, PA, April 1999.

[4] O. Khatib, O. Brock, K.-S. Chang, D. Ruspini, L. Sentis, and S. Viji, "Human-centered robotics and interactive haptic simulation," Int'l. J. of Robotics Research, vol. 23, no. 2, pp. 167-178, February 2004.
[5] O. J. Rosch, K. Schilling, and H. Roth, "Haptic interfaces for the remote control of mobile robots," Control Engineering Practice, vol. 10, no. 11, pp. 1309-1313, November 2002.

[6] R. Ott, M. Gutierrez, D. Thalmann, and F. Vexo, "VR haptic interfaces for teleoperation: an evaluation study," in Proc. IEEE Intelligent Vehicles Symp. IV, Las Vegas, NV, June 2005.

[7] J. B. Park, B. H. Lee, and M. S. Kim, "Remote control of a mobile robot using distance-based reflective force," in Proc. IEEE Int'l. Conf. on Robotics and Automation, Taipei, Taiwan, September 14-19 2003, pp. 3415-3420.

[8] D. S. Ryu, C. H. Cho, M. S. Kim, and J. B. Song, "Design of a 6-DOF haptic master for tele-operating a mobile manipulator," in Proc. IEEE Int'l. Conf. on Robotics and Automation, Taipei, Taiwan, September 14-19 2003, pp. 3243-3248.

[9] N. Diolaiti and C. Melchiorri, "Tele-operation of a mobile robot through haptic feedback," in IEEE Int'l. Workshop on Haptic Virtual Environments and Their Applications (HAVE 2002), Ottawa, Ontario, Canada, November 17-18 2002.

[10] N. Diolaiti and claudio Melchiorri, "Haptic tele-operation of a mobile robot," in Proc. 7th IFAC Symp. on Robot Control (SYROCO 2003), Wroclaw, Poland, September 1-3 2003.

[11] S. Lee, G. S. Sukhatme, G. J. Kim, and C.-M. Park, "Haptic teleoperation of a mobile robot: a user study," Presence, vol. 14, no. 3, pp. 345-365, June 2005

[12] I. Elhajj, H. Hummert, and N. Xi, "Real-time bilateral control of Internet-based teleoperation," in Proc. 3rd. World Congress on Intelligent Control and Automation, Hefei, China, June 28-July 2 2000, pp. 3761-3766.

[13] I. Elhajj, H. Hummert, N. Xi, Y.-H. Liu, and W. J. Li, "Sychronization and control of supermedia transmission via the Internet," in Proc. of 2001 Int'l. Symp. on Intelligent Multimedia, Video, and Speech Processing, Hong Kong, China, May 24 2001, pp. 320-323.

[14] J. Lim, J. Ko, and J. Lee, "Internet-based teleoperation of a mobile robot with force-reflection," in Proc. IEEE Conf. on Control Applications (CCA), Istanbul, Turkey, June 2003, pp. 23-25.

[15] D. Lee and M. W. Spong, "Bilateral teleoperation of a wheeled mobile robot over delayed communication network," in Proc. IEEE Int'l. Conf. on Robotics and Automation, Orlando, FL, May 15-19 2006

[16] U. Saranli, M. Buehler, and D. E. Koditschek, "RHex: a simple and highly mobile robot," Int'l. J. of Robotics Research, vol. 20, no. 7, pp. 616-631, July 2001.

[17] U. Saranli, A. Rizzi, and D. E. Koditschek, "Multi-point contact models for dynamic self-righting of a hexapod robot," in Proc. 6th Int'l. Workshop on the Algorithmic Foundations of Robotics (WAFR '04), July 2004, pp. 75-90.

[18] R. H. LaMotte and M. A. Srinivasan, Information Processing in the Somatosensory System, ser. Wenner-Gren International Symposium. MacMillan Press, 1990, ch. Surface microgeometry: Tactile perception and neural coding.

[19] J. E. Colgate, M. C. Stanley, and J. M. Brown, "Issues in the haptic display of tool use," in Proc. IEEE/RSJ Int'l. Conf. on Intelligent Robots and Systems (IROS), 1995, pp. 140-145. 